\title{
The Mechanism of Coronary Artery Calcification in Centrally Obese Non-Diabetic Men: Study on The Interaction of Leptin, Free Leptin Index, Adiponectin, hs-C Reactive Protein, Bone Morphogenetic Protein-2 and Matrix Gla Protein
}

\author{
Antonia Anna Lukito', Syakib Bakri², Peter Kabo², Andi Wijaya ${ }^{2,3}$ \\ ${ }^{1}$ Faculty of Medicine, Universitas Pelita Harapan/Siloam Hospitals Lippo Village, Tangerang, Indonesia \\ ${ }^{2}$ Faculty of Medicine, Universitas Hasanuddin, Makassar, Indonesia \\ ${ }^{3}$ Prodia Clinical Laboratory, Bandung, Indonesia
}

Background: The calcium in the artery was thought to be the result of the imbalance or dysregulation of the promoter and inhibitor cytokines influenced by various subclinical and clinical conditions. This study aimed to investigate the interaction between central obesity, as an early subclinical condition, also known as a chronic low grade inflammation condition and coronary artery calcium (CAC) in non-diabetic population including the underlying pathomechanisms of a CAC in the early stage of atherosclerosis.

Materials and Methods: This was a cross-sectional pathway analysis study enrolling 60 central obesity non-diabetic men that underwent coronary calcium score scan, anthropometrics and biomarker assays.

Results: There was a positive correlation between increasing free leptin index/adiponectin (FLI/A) ratio and CAC ( $r=0.297$; $p<0.05)$. There was a positive correlation between increasing FLI/A ratio and plasma high sensitive C-reactive protein (hsCRP) $(r=0.318 ; p<0.05)$. Plasma hs-CRP and bone morphogenetic protein-2 (BMP-2)-matrix gla protein (MGP) dysregulation were positively correlated $(r=0.221 ; p<0.05)$ after adjusted to risk factors including insulin resistance, hypertension, age, and dyslipidemia.

Conclusion: The study found that one of the pathways involved in CAC in the centrally obese non-diabetic male is might be due to an increase of free leptin and decrease of adiponectin. The free leptin and adiponection ratio also increased hs-CRP, which partially correlated to the dysregulation of BMP-2 and MGP.

Keywords: coronary artery calcification, central obesity, adipokines, bone regulator protein, pathomechanism

\section{Introduction}

The mechanism involved in atherosclerosis consists of local vascular injury, inflammation, oxidative stress and vascular calcification. ${ }^{1}$ Vascular calcification at the early stage of atherosclerosis was demonstrated in recent studies and are associated with cardiovascular mortality and morbidity. ${ }^{2,3}$ The degree of calcification was associated

Date of submission: July 27, 2019

Last Revised: October 17, 2019

Accepted for publication: October 22, 2019

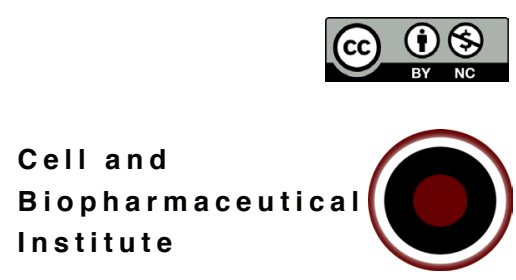

Corresponding Author:

Antonia Anna Lukito

Faculty of Medicine, Universitas Pelita Harapan

J. MH. Thamrin Boulevard 1100

Karawaci, Tangerang, Banten 15811, Indonesia

e-mail: lukito_ant@yahoo.com 
with local vascular inflammation and progression of atherosclerosis. ${ }^{1,4}$

Adipocyte and macrophages in fat tissues secrete cytokines that are involved in osteogenic mineralization of atherosclerotic process which in turn stimulates the secretion of hepatic C-reactive protein (CRP), was thought to be central in the relationship between inflammation and coronary calcification..$^{5-8}$ Vascular calcification is a result of the imbalance between factors that promote calcification and mineralization inhibitor, these are bone morphogenetic protein-2 (BMP-2), leptin, oxidative stress, hyperphosphatemia, hyperparathyroidism, and vitamin D. While factors that inhibit mineralization includes matrix-carboxyglutamic acid gla protein (MGP), fetuin, adiponectin, and osteopontin. ${ }^{9}$

Adiponectin is an anti-inflammatory and antiatherogenic substance which secretion is decreased in obesity. ${ }^{10}$ Plasma adiponectin concentration was independently associated with coronary artery calcium (CAC) score. Increased circulating leptin concentration is a marker of leptin resistance and is commonly found in obese patients. ${ }^{11}$

Free leptin index (FLI) which is the ratio of leptin and soluble leptin receptor (SLR), is thought to be a more accurate marker of leptin function. ${ }^{12}$ SLR is the main binding protein for leptin in human blood, and therefore is capable of pivotally modulating its bioavailability. ${ }^{13}$ Plasma leptin:adiponectin concentration ratio (L:A ratio) was thought to be an atherosclerotic index in type 2 DM patients and also a stronger indicator of carotid intimal media thickness (IMT). ${ }^{14-16}$ However, the data regarding the role of FLI/adiponectin (FLI/A) ratio is scarce. CAC score or degree of coronary calcification is an independent predictor for coronary artery disease. ${ }^{17}$

BMP-2 promotes calcification because it is a powerful osteogenic protein needed for differentiation of osteoblast and bone formation is also central in the mechanism of vascular calcification. ${ }^{18}$ MGP is known as cartilage and vascular calcification inhibitor by regulating BMP-2. ${ }^{19}$

The mechanism of vascular calcification in nondiabetic population with central obesity remained elusive. This study aimed to investigate the interaction between central obesity and CAC in non-diabetic population including the underlying pathomechanism. The pathomechanism that will be studied includes marker of adipokine dysfunction (leptin, free leptin index, adiponectin), inflammation (hsCRP), and bone regulator protein (BMP-2 and MGP).
We decided to exclude female patients from this study because the prevalence of coronary calcification and CAC score were lower in female compared to male ${ }^{20}$, a higher leptin concentration in female, and lower adiponectin and SLR concentration in female compared to male counterpart. ${ }^{21,22}$

\section{Materials and methods}

\section{Subject Population}

In this observational cross-sectional study, patients who were referred to the radiology department of Siloam Hospitals Lippo Village, Tangerang, Indonesia for Calcium score assessment using Dual Source 64-slice Computed Tomography scan during 2010-2011 period were consecutively enrolled. Our study was approved by the Institutional ethics committee at Faculty of medicine, Hasanuddin University, Makassar, Indonesia (Ethical approval recommendation letter No.2062/H04.8.4.5.31/ PP36-KOMETIK/2010).

Inclusion criteria were as follows: (1) male $>45$ years old; (2) waist circumference $>90 \mathrm{~cm}$; (3) no prior history of diabetes, chronic kidney disease, or transaminitis; (4) CAC score $>10 ;(5)$ no prior history of coronary stent or coronary bypass grafting; (6) no current anticoagulant regiment; (7) absence of acute inflammatory states; and (8) willing to participate in study. All patients who fulfill the inclusion criteria were enrolled. The calculated sample size was 52.78 subjects, and rounded up to 60 subjects.

\section{Dual Source 64 Slice Computed Tomography}

CAC scoring of the subjects were performed on Dual Source 64 Slice Computed tomography (Siemens Healthineers, Erlangen, Germany) with prospective electrocardiographic gating method, and subjects holding their breath for 7 seconds, by sequential data acquisition with slice thickness of $3 \mathrm{~mm}$.

Table 1. Subjects' characteristic.

\begin{tabular}{lc}
\hline \multicolumn{1}{c}{$\begin{array}{c}\text { Subjects Characteristics } \\
(\mathbf{n}=\mathbf{6 0})\end{array}$} & $\mathbf{n}(\mathbf{\%})$ \\
\hline Smoker & $9(15.0 \%)$ \\
Exercise & $34(56.7 \%)$ \\
Hypertension & $36(60.0 \%)$ \\
Hypertension therapy & $25(41.7 \%)$ \\
Statin therapy & $22(36.7 \%)$ \\
\hline
\end{tabular}


Table 2. Clinical variables of subjects.

\begin{tabular}{|c|c|c|c|c|}
\hline $\begin{array}{l}\text { Subjects Characteristics } \\
\qquad(\mathrm{n}=60)\end{array}$ & Minimum & Maximum & Median & Mean \pm SD \\
\hline Age (years old) & 45 & 70 & 55 & $55.20 \pm 6.51$ \\
\hline Height (cm) & 155 & 180 & 167 & $167.0 \pm 5.9$ \\
\hline Weight (kg) & 62 & 112 & 80 & $80.3 \pm 10.8$ \\
\hline BMI $\left(\mathrm{kg} / \mathrm{m}^{2}\right)$ & 23.2 & 37.5 & 28.1 & $28.8 \pm 3.3$ \\
\hline $\mathrm{NC}(\mathrm{cm})$ & 35 & 49.5 & 40 & $40.2 \pm 2.9$ \\
\hline $\mathrm{AC}(\mathrm{cm})$ & 90 & 125 & 99 & $101.0 \pm 7.9$ \\
\hline SBP (mmHg) & 100 & 183 & 130 & $129.3 \pm 14.8$ \\
\hline DBP (mmHg ) & 60 & 100 & 80 & $82.0 \pm 8.6$ \\
\hline PP (mmHg ) & 30 & 103 & 42.5 & $47.3 \pm 12.3$ \\
\hline MAP (mmHg ) & 80 & 120 & 96.7 & $97.7 \pm 9.4$ \\
\hline FBG (mg/dL) & 81 & 123 & 98 & $99.4 \pm 10.5$ \\
\hline HOMA IR & 0.46 & 6.42 & 2.7 & $2.89 \pm 1.45$ \\
\hline TC (mg/dL) & 97 & 286 & 206.5 & $207.9 \pm 39.5$ \\
\hline $\mathrm{HDL}(\mathrm{mg} / \mathrm{dL})$ & 26 & 61 & 41.5 & $42.2 \pm 7.7$ \\
\hline LDL (mg/dL) & 42 & 205 & 130.5 & $131.2 \pm 36.6$ \\
\hline TG (mg/dL) & 39 & 396 & 131 & $146 \pm 74$ \\
\hline CAC (Agatston Unit) & 11 & 770 & 117.2 & $173.6 \pm 177.8$ \\
\hline Adiponectin (ng/mL) & 1218 & 7311 & 3356.4 & $3627.9 \pm 1256.7$ \\
\hline Leptin (ng/mL) & 2.2 & 31.1 & 9.4 & $10.8 \pm 6.4$ \\
\hline SLR (ng/mL) & 11.5 & 34.8 & 21.53 & $21.94 \pm 5.5$ \\
\hline FLI & 0.1 & 1.8 & 0.5 & $0.6 \pm 0.4$ \\
\hline Hs-CRP (mg/dL) & 0.2 & 9.6 & 1.5 & $2.3 \pm 2.1$ \\
\hline BMP-2 (pg/dL) & 142 & 4095 & 1796 & $1736.6 \pm 1015.7$ \\
\hline MGP (pg/dL) & 56941 & 198915.5 & 91356.1 & $100407.0 \pm 25916.7$ \\
\hline
\end{tabular}

BMI: body mass index; NC: neck circumference; AC: abdominal circumference; SBP: systolic blood pressure; DBP: diastolic blood pressure; PP: pulse pressure; MAP: mean arterial pressure; FBG: fasting blood glucose; HOMA IR: homeostasis model assessment of insulin resistance; TC: total cholesterol; HDL: high density lipoprotein cholesterol; LDL: low density lipoprotein; TG: triglyceride; CAC: coronary artery calcium; SLR: soluble leptin receptor; FLI: free leptin index; hs-CRP: high sensitive C-reactive protein; BMP2: bone morphogenetic protein; MGP: matrix gla protein.

\section{CAC Scoring}

CAC scoring was calculated by modified Agatston score with detection threshold of $130 \mathrm{HU}$ (Hounsfield Unit), performed using semi-automatical software (Syngo Calcium Scoring, Siemens Medical Solutions, Erlangen, Germany). Each data was determined by a certified radiology technician and a certified radiologist physician.

\section{Biomarkers Assay}

Serum level of adiponectin, leptin, SLR, BMP-2 were measured using enzyme-linked immunosorbent assay (ELISA) method, while serum level of MGP was using quantitative enzyme immunoassay (EIA) method. Fasting insulin concentration was measured using immunochemiluminescent method. Laboratory tests of 
the subjects were done by Prodia Laboratories (PT Prodia Widyahusada, Jakarta, Indonesia).

FLI is defined as a ratio of plasma leptin over plasma SLR levels, with cut-off point derived from median, the normal values of adiponectin is $3-30 \mu \mathrm{g} / \mathrm{mL}$, leptin $2-8 \mu \mathrm{g} /$ $\mathrm{mL}$, SLR $81.2 \pm 143.2 \mathrm{ng} / \mathrm{mL}$, and the cut-off points of BPM2 and MGP are derived from median.

\section{Statistical Analysis}

Data analyses using univariate, bivariate and multivariate approaches were performed. Results were narrated and illustrated using tables and figures. Statistical tests that were performed includes: (1) descriptive analysis using univariate methods to compute minimum and maximum values, median, mean and standard of deviation; (2) normalcy test; (3) Pearson and Spearman correlation tests; (4) partial correlation test of parametric and non-parametric values; (5) cross-tabulations. All analyses were performed with the IBM SPSS version 15 on windows (IBM Inc, Armonk, NY, USA).

\section{Results}

There were 60 subjects that fulfilled the inclusion criteria. The characteristics of these subjects were summarized in Table 1 and Table 2. Mean CAC score was $173.6 \pm 177.8$ and mean FLI was $0.6 \pm 0.4$.
Table 3. Correlation between adipokine, inflammation, bone protein dysregulation and CAC.

\begin{tabular}{lcc}
\hline \multicolumn{1}{c}{ Variable } & \multicolumn{2}{c}{ CAC (n=60) } \\
\cline { 2 - 3 } & $\begin{array}{c}\text { Correlation } \\
\text { Coefficient (r) }\end{array}$ & $\boldsymbol{p}$-value \\
\hline FLI/Adiponectin & 0.297 & $<0.05$ \\
Hs-CRP & 0.077 & $>0.05$ \\
BMP-2 and MGP dysregulation & 0.015 & $>0.05$ \\
\hline
\end{tabular}

CAC: coronary artery calcium; SLR: soluble leptin receptor; FLI: free leptin index; hs-CRP: high sensitive C-reactive protein; BMP-2: bone morphogenetic protein; MGP: matrix gla protein. Tested with Spearman one-tailed bivariate analysis.

There was a positive correlation between increasing $\mathrm{FLI} / \mathrm{A}$ ratio and CAC $(r=0.297 ; p<0.05)$. However, there was no significant correlation between plasma hs-CRP and BMP-2-MGP dysregulation with CAC (Table 3, Figure 1). Plasma hs-CRP and BMP-2-MGP dysregulation were positively correlated $(r=0.221 ; p<0.05)$ after adjustment of age, hypertension, insulin resistance and dyslipidemia and there was no significant correlation between FLI/A ratio and BMP-2-MGP dysregulation. There was a significant positive correlation between FLI/A ratio and hs-CRP $(r=0.318 ; p<0.05)$.

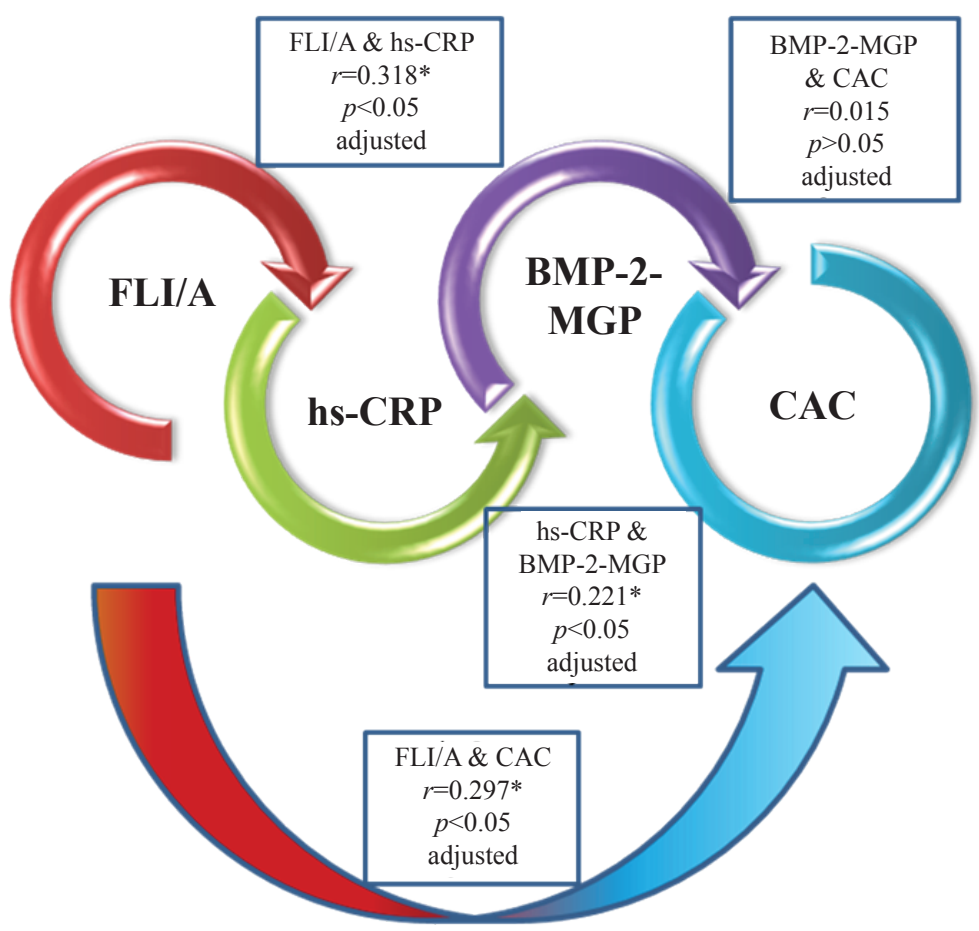

Figure 1. Correlation between variables in this study. FLI: free leptin index; A: adiponectin; hs-CRP: high sensitivity C-reactive protein; BMP-2: bone morphogenetic protein-2; MGP: matrix gla-protein; CAC: coronary artery calcium. Tested with partial bivariate analysis Spearman one-tailed; *Significant correlation at $p<0,05$; Adjusted to insulin resistance, age, hypertension, and dyslipidemia. 


\section{Discussion}

A positive correlation between $\mathrm{FLI} / \mathrm{A}$ ratio and CAC in this study is in agreement with a cohort study that reports an inverse relationship between adiponectin and progression of CAC in both type 1 diabetic and non-diabetics. ${ }^{23}$ Leptin regulates osteoblastic differentiation, vascular cell calcification, and the presence of leptin receptor on the calcified vascular cells. ${ }^{24}$ This might also stem from the fact that leptin triggers vascular inflammation by activating immune cells, increasing CRP and oxidative stress, and directly changed vascular conditions by initiating proliferation and migration of smooth muscle cells causing calcification and reducing the compliance of vessel walls. ${ }^{25}$ Obesity is a low-grade chronic inflammatory condition caused by a change in adipocyte and macrophage function resulting in adipokine hypersecretion which is an atherogenic, pro-inflammatory, and pro-diabetic followed by reduced production of anti-inflammatory adiponectin. ${ }^{26}$ Another important finding is that FLI/A ratio and hs-CRP were positively correlated in our study, adipokine was associated with chronic low-grade inflammation. ${ }^{27} \mathrm{~A}$ study showed that high serum leptin increased the coronary artery endothelial production of CRP suggesting its pro-atherogenicity. ${ }^{28}$ There was an inverse relationship between adiponectin and plasma CRP in both plasma and adipocytes. ${ }^{29}$

Our study showed that there is no direct correlation between increased FLI/A ratio and dysregulation of BMP-2MGP balance. A positive correlation was found between hsCRP as inflammatory markers and dysregulation of BMP-2MGP balance after adjusted to risk factors including insulin resistance, hypertension, age, and dyslipidemia. This result may be due to BMP-2 induced by inflammation. ${ }^{30}$

No significant correlation between either hs-CRP or dysregulation of BMP-2-MGP balance with CAC score in this study might be due to the dynamic biological phenomenon between promoting and inhibitory factors affecting CAC. Hence, it is difficult to get a static and absolute variable in one period. A proposed mechanism of CAC caused by central obesity is shown in Figure 2.

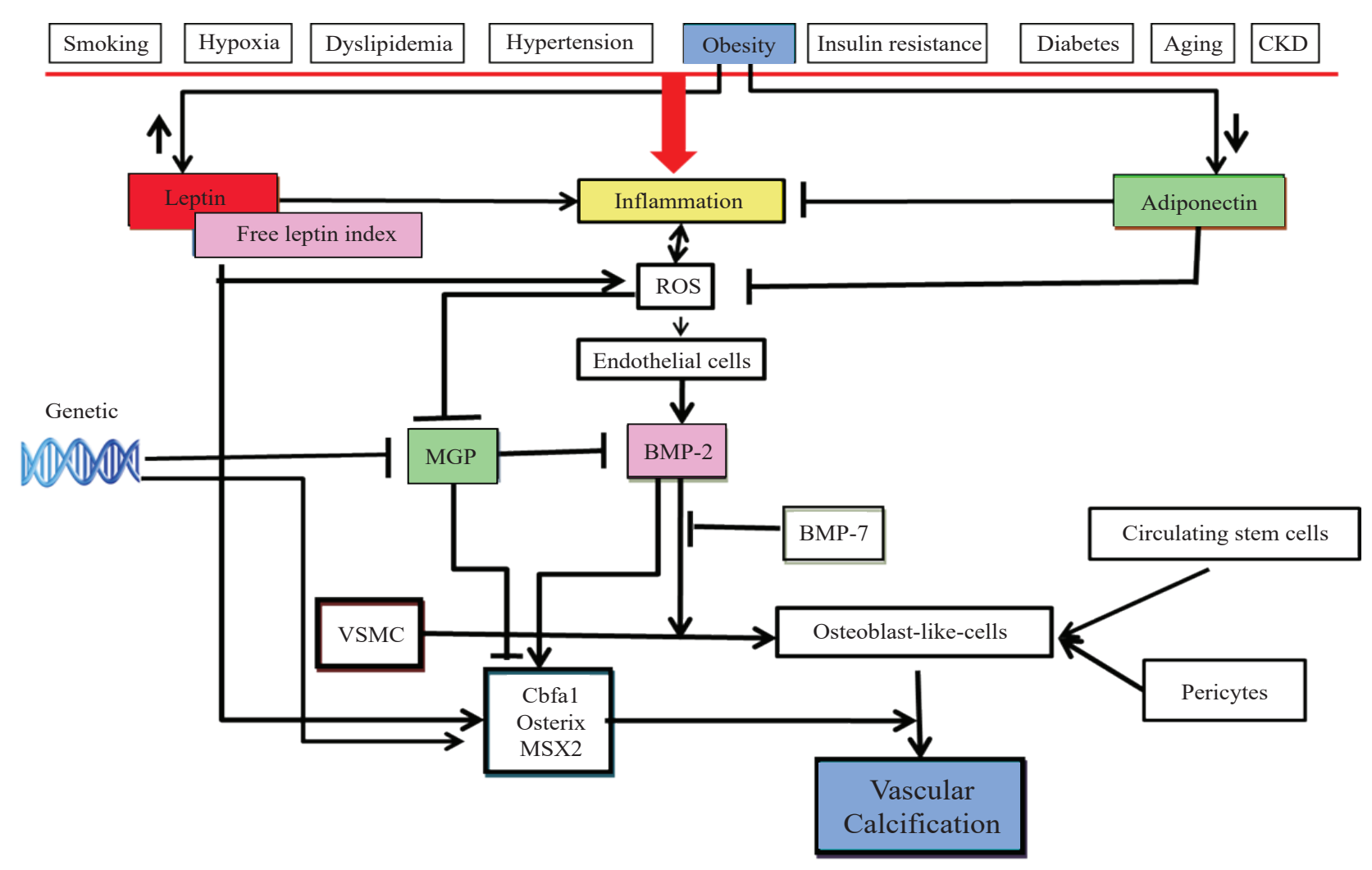

Figure 2. Proposed mechanism of CAC caused by central obesity. 1Cbfa1: core-binding factor alpha-1; BMP: bone morphogenetic protein; MGP: matrix gla protein; MSX2: homeobox protein MSX-2; Ox-LDL: oxidized low-density lipoprotein; ROS: reactive oxygen species; VSMC: vascular smooth muscle; CKD: chronic kidney disease. 


\section{Conclusion}

In this study, we found the pathways involved in CAC in the centrally obese non-diabetic males were the increase of free leptin and a decrease of adiponectin, which correlated with the degree of coronary artery calcification. The increased of free leptin and adiponectin ratio was also leading to the increase of low grade inflammation hs-CRP, which eventually increases the promoter of calcification (BMP2) and reduces the inhibitor of calcification (MGP). These dynamic pathways were influenced by risk factors such as insulin resistance, age, hypertension and dyslipidemia.

\section{References}

1. Mazzini MJ, Schulze PC. Proatherogenic pathways leading to vascular calcification. Eur J Radiol. 2006; 57(3): 384-9.

2. Giachelli CM. Vascular calcification mechanisms. J Am Soc Nephrol. 2004; 15(12): 2959-64.

3. Raggi P, Boulay A, Chasan-Taber S, Amin N, Dillon M, Burke SK, et al. Cardiac calcification in adult hemodialysis patients. A link between end-stage renal disease and cardiovascular disease? J Am Coll Cardiol. 2002; 39(4): 695-701.

4. Belovici MI, Pandele GI. Arterial media calcification in patients with type 2 diabetes mellitus. Rev Med Chir Soc Med Nat Iasi. 2008; 112(1): 21-34.

5. Wang Z, Nakayama T. Inflammation, a link between obesity and cardiovascular disease. Mediators Inflamm. 2010; 2010: 1-17. doi: $10.1155 / 2010 / 535918$.

6. Rajala MW, Scherer PE. Minireview: the adipocyte — at the crossroads of energy homeostasis, inflammation, and atherosclerosis. Endocrinology. 2003; 144(9): 3765-73.

7. Shao JS, Cheng SL, Sadhu J, Towler DA. Inflammation and the osteogenic regulation of vascular calcification. Hypertension. 2010; 55(3): 579-92.

8. Tieu BC, Lee C, Sun H, Lejeune W, Recinos A 3rd, Ju X, et al. An adventitial IL-6/MCP1 amplification loop accelerates macrophagemediated vascular inflammation leading to aortic dissection in mice. J Clin Invest. 2009; 119(12): 3637-51.

9. Johnson RC, Leopold JA, Loscalzo J. Vascular calcification. Circ Res. 2006; 99(10): 1044-59.

10. Hattori Y, Nakano Y, Hattori S, Tomizawa A, Inukai K, Kasai K. High molecular weight adiponectin activates AMPK and suppresses cytokine-induced NF- $\mathrm{kB}$ activation in vascular endothelial cells. FEBS Lett. 2008; 582(12): 1719-24.

11. Martin SS, Qasim A, Reilly MP. Leptin resistance: a possible interface of inflammation and metabolism in obesity-related cardiovascular disease. J Am Coll Cardiol. 2008; 52(15): 1201-10.

12. Thomopoulos C, Papadopoulos DP, Papazachou O, Bratsas A, Massias S, Anastasiadis G, et al. Free leptin is associated with masked hypertension in nonobese subjects. Hypertension. 2009; 53(6): 965-972.

13. Schaab M, Kratzsch J. The soluble leptin receptor. Best Pract Res Clin Endocrinol Metab. 2015; 29(5): 661-70.
14. Inoue M, Maehata E, Yano M, Taniyama M, Suzuki S. Correlation between the adiponectin-leptin ratio and parameters of insulin resistance in patients with type 2 diabetes. Metabolism. 2005; 54(3): 281-6.

15. Inoue M, Yano M, Yamakado M, Maehata E, Suzuki S. Relationship between the adiponectin-leptin ratio and parameters of insulin resistance in subjects without hyperglycemia. Metabolism. 2006; 55(9): 1248-54.

16. Norata GD, Raselli S, Grigore L, Garlaschelli K, Dozio E, Magni P, et al. Leptin:adiponectin ratio is an independent predictor of intima media thickness of the common carotid artery. Stroke. 2007; 38(10): 2844-6.

17. Schmermund A, Baumgart D, Görge G, Grönemeyer D, Seibel R, Bailey KR, et al. Measuring the effect of risk factors on coronary atherosclerosis: coronary calcium score versus angiographic disease severity. J Am Coll Cardiol. 1998; 31(6): 1267-73.

18. Li X, Yang HY, Giachelli CM. BMP-2 promotes phosphate uptake, phenotypic modulation, and calcification of human vascular smooth muscle cells. Atherosclerosis. 2008; 199(2): 271-7.

19. Zebboudj AF, Imura M, Boström K. Matrix GLA protein, a regulatory protein for bone morphogenetic protein-2. J Biol Chem. 2002; 277(6): 4388-94.

20. Raggi P, Shaw LJ, Berman DS, Callister TQ. Gender-based differences in the prognostic value of coronary calcification. J Womens Health (Larchmt). 2004; 13(3): 273-83.

21. Ogier V, Ziegler O, Méjean L, Nicolas JP, Stricker-Krongrad A. Obesity is associated with decreasing levels of the circulating soluble leptin receptor in humans. Int J Obes Relat Metab Disord. 2002; 26(4): 496-503.

22. Kaser S, Tatarczyk T, Stadlmayr A, Ciardi C, Ress C, Tschoner A, et al. Effect of obesity and insulin sensitivity on adiponectin isoform distribution. Eur J Clin Invest. 2008; 38(11): 827-34.

23. Maahs DM, Ogden LG, Kinney GL, Wadwa P, Snell-Bergeon JK, Dabelea D, et al. Low plasma adiponectin levels predict progression of coronary artery calcification. Circulation. 2005; 111(6): 747-53.

24. Parhami F, Basseri B, Hwang J, Tintut Y, Demer LL. High-density lipoprotein regulates calcification of vascular cells. Circ Res. 2002; 91(7): 570-6.

25. Dubey L, Hesong Z. Role of leptin in atherogenesis. Exp Clin Cardiol. 2006; 11(4): 269-75.

26. Hajer GR, van Haeften TW, Visseren FLJ. Adipose tissue dysfunction in obesity, diabetes, and vascular diseases. Eur Heart J. 2008; 29(24): 2959-71.

27. Ikeoka D, Mader JK, Pieber TR. Adipose tissue, inflammation and cardiovascular disease. Rev Assoc Med Bras. 2010; 56(1): 11621.

28. Singh P, Hoffmann M, Wolk R, Shamsuzzaman ASM, Somers VK. Leptin induces C-reactive protein expression in vascular endothelial cells. Arterioscler Thromb Vasc Biol. 2007; 27(9): e302-7.

29. Ouchi N, Kihara S, Funahashi T, Nakamura T, Nishida M, Kumada M, et al. Reciprocal association of C-reactive protein with adiponectin in blood stream and adipose tissue. Circulation. 2003; 107(5): 671-4.

30. Shao JS, Cai J, Towler DA. Molecular mechanisms of vascular calcification. Arterioscler Thromb Vasc Biol. 2006; 26(7): 142330 . 